\title{
openheart Multicentre analysis of current ST-elevation myocardial infarction acute care pathways
}

\author{
Joppe Tra, ${ }^{1}$ Carolien de Blok, ${ }^{2}$ Ineke van der Wulp, ${ }^{1}$ Martine C de Bruijne, ${ }^{1}$ \\ Cordula Wagner ${ }^{1,3}$
}

To cite: Tra J, de Blok C, van der Wulp I, et al. Multicentre analysis of current ST-elevation myocardial infarction acute care pathways. Open Heart 2017; 4:e000458. doi:10.1136/openhrt-2016000458

- Additional material is available. To view please visit the journal online (http://dx. doi.org/10.1136/openhrt2016-000458)

Received 27 July 2016 Revised 23 November 2016 Accepted 20 December 2016

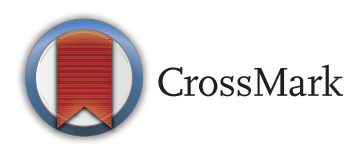

${ }^{1}$ Department of Occupational and Public Health, EMGO+NU University Medical Center, Amsterdam, The Netherlands ${ }^{2}$ Faculty of Economics and Business, Department of Operations, University of Groningen, Groningen, The Netherlands

${ }^{3}$ The Netherlands Institute of Health Services Research (NIVEL), Utrecht,

The Netherlands

Correspondence to

Joppe Tra;

joppetra@gmail.com

\section{ABSTRACT}

Background: Rapid reperfusion with percutaneous coronary intervention $(\mathrm{PCl})$ is vital for patients with ST segment elevation myocardial infarction (STEMI). However, the guideline-recommended time targets are regularly exceeded. The goal of this study was to gain insight into how Dutch PCl centres try to achieve these time targets by comparing their care processes with one another and with the European guidelinerecommended process. In addition, accelerating factors perceived by care providers were identified.

Methods: In this multiple case study, interviews with STEMI care providers were conducted, transcribed and used to create process descriptions per centre. Analyses consisted of within-case and between-case analyses of the processes. Accelerating factors were identified by means of open and axial coding.

Results: In total, 28 interviews were conducted in six $\mathrm{PCl}$ centres. The centres differed from the guidelinerecommended process on, for example, additional, unavoidable patient routings and monitoring delays, and from one another on the communication of diagnostic information (eg, transmitting all, only ambiguous or no ECGs) and catheterisation room preparation. These differences indicated diverging choices to maintain a balance between speed and diagnostic accuracy. Factors perceived by care providers as accelerating the process included trust in the tentative diagnosis, and avoiding unnecessary intercaregiver consultations. The combination of processes and accelerating factors were summarised in a model.

Conclusions: Numerous differences in processes between $\mathrm{PCl}$ centres were identified. Several timesaving strategies were applied by $\mathrm{PCl}$ centres, however, in different configurations. To further improve the care for patients with STEMI, best practices can be shared between centres and countries.

\section{INTRODUCTION}

Rapid reperfusion treatment increases the chances of survival for patients with ST segment elevation myocardial infarction (STEMI). ${ }^{1} \quad 2$ Consequently, international STEMI care guidelines recommend limiting the time to treatment with percutaneous

\section{KEY QUESTIONS}

What is already known about this subject?

- Achieving the guideline recommended treatment delays for patients with ST segment elevation myocardial infarction (STEMI) going for percutaneous coronary intervention (PCl) is difficult. Several studies have investigated the association of arrival and patient characteristics with treatment delays, but few studies have compared acute STEMI care processes between $\mathrm{PCl}$ centres.

\section{What does this study add?}

- Numerous differences in processes between PCI centres were identified, indicating diverging choices to maintain a balance between speed and diagnostic accuracy. Several factors accelerating the process were identified, which can be used for further improvement.

How might this impact on clinical practice?

- By sharing best practices and/or comparing regular practice with the results of this study, treatment delays can be further reduced, potentially resulting in improved patient outcomes.

coronary intervention (PCI) to a maximum of 90 min from first (para)medical contact. ${ }^{3} 4$ However, previous studies reported that attaining these time targets is difficult. ${ }^{5}$

In the European Society of Cardiology guidelines, a model of care has been described to treat patients within 90 min after first (para)medical contact. In this description, patients contact the emergency medical services through a central telephone number. In case of a suspected myocardial infarction, the emergency medical services dispatch an ambulance. In the ambulance, an ECG is recorded and interpreted by trained paramedics, and/or the ECG is transferred for a teleconsultation with a cardiology centre. Triage, diagnosis and emergency treatment are all performed in the prehospital phase. The cardiology centres, with 24/7 PCI services, 
cooperate with other hospitals in the region and with the emergency medical services through clear geographic boundaries, shared protocols and bypassing of non-PCI hospitals. While the patient is en route to the hospital, the catheterisation laboratory staff are called and the room is prepared. On arrival, the patient bypasses the emergency department and intensive coronary care unit and is immediately transferred to the catheterisation laboratory. The treatment delays are measured and used to improve the process of care.

Despite the aforementioned guideline-recommended process, the recommended maximal treatment delay of $90 \mathrm{~min}$ is still exceeded for a considerable number of patients in the Netherlands. ${ }^{6}$ Although treating all patients within the recommended time targets is unlikely, there appears room for improvement. In addition to a well-designed process, hospital-related, patientrelated and physician-related factors including annual PCI volume and time of presentation appear to influence the speed of the care process as well. ${ }^{7}$ Therefore, further optimising the logistic processes and taking into account accelerating factors may reduce the treatment delay and subsequently lower the patients' risk of adverse cardiac events. ${ }^{8}$ Therefore, the primary objective of this study was to explore how PCI centres in the Netherlands differed in their logistic processes from the European guideline-recommended process and from one another. The secondary objective was to identify factors potentially accelerating the process.

\section{METHODS}

\section{Design}

Since quantitative information about the delays in the acute STEMI care process is lacking in many countries including the Netherlands, ${ }^{9}{ }^{10}$ the study was performed in a multiple case study design, using PCI centres as cases. This type of design is well suited for exploring and comparing complex processes in a real-life context. ${ }^{11}$

\section{Setting}

The study was conducted in the Netherlands, a country of $\sim 34000 \mathrm{~km}^{2}$ where annually more than 11000 patients are treated with primary PCI in 30 PCI centres. ${ }^{12}$ Owing to an efficient geographical spread of PCI centres in the Netherlands, PCI is the preferred reperfusion therapy for all patients with STEMI who present within 12 hours of symptom onset. Therefore, timely provision of fibrinolysis was not taken into account in this study.

To access a PCI centre, patients may take different routes. In the Dutch healthcare system, the general practitioner has a gatekeeping role, meaning that referral from a general practitioner is required to see a hospital physician. Exceptions are made for medical emergencies such as STEMI, but some patients with symptoms of STEMI contact their general practitioner or general practitioners' after hours office. In their guidelines for acute coronary syndrome, general practitioners are recommended to call the emergency medical services and perform an anamnesis and physical examination. ${ }^{13}$

Alternatively, patients can contact the emergency medical services in the Netherlands directly by dialling the national emergency number (112). If indicated by the triage system, an operator of 1 out of 25 selfdispatching regional ambulance services sends an ambulance to the patient. Ambulances are staffed by a driver and by a nurse licensed to administer medical treatment at an advanced life support level. ${ }^{14}$ All ambulance services work according to a national ambulance protocol which allows for regional adaptations in cooperation with the PCI centres. ${ }^{15}$ This protocol describes transporting patients with a (tentative) STEMI diagnosis directly to the nearest PCI centre. ${ }^{16}$ As a result of these national structures, the treatment delay in this study was defined as the period from first (para)medical contact in person to the PCI procedure. In addition, the care process was defined as all statements pertaining to individual tasks and responsibilities that contributed to getting the patient to a prepared catheterisation room.

\section{Selection of PCI centres and participants}

In total, seven hospitals providing primary PCI 24/7 were invited to participate in this study after participating in a previous study, for which they were selected using a multistage random selection procedure. ${ }^{17}$ These PCI centres differed among other things in ambulance region, annual PCI volume and type of hospital (University vs non-University teaching). At each centre, an interventional cardiologist specialised in acute coronary syndromes was invited by email for an interview. In the interviews, cardiologists were asked to provide contact details of one person from each profession involved in acute STEMI care at the hospital or at the emergency medical services. These professionals were subsequently approached by email to participate in the study. Additionally, for verification of the information given about the additional prehospital care processes, cardiologists from referring hospitals and general practitioners were invited for participation through the interventional cardiologist.

\section{Data collection}

Data were collected by means of one-on-one semistructured interviews at the workplace or home of the participant. The interviews were conducted with a topic list based on the European guideline-recommended process. ${ }^{3}$ The topic list was tested in two pilot interviews and adjusted accordingly, resulting in a final topic list (see online supplementary table S1). Each interview started with a grand tour question in which cardiologists were asked to describe the care process from symptom onset to reperfusion for patients with STEMI going for primary PCI. Subsequent questions were related to the steps in the care process described by the participant, the role of other care providers in the process, prior 
quality improvement efforts and monitoring of the guideline-recommended time targets.

The interviews were performed by one interviewer, trained in qualitative interviewing (JT), between May 2013 and February 2014, were audio-recorded and transcribed verbatim using the computer program F4 2012 (V.5.2, Dr Dresing and Pehl GmbH—audiotranskription.de).

\section{Data analyses}

In analysing the process of STEMI care, the data reduction strategy of Miles and Huberman ${ }^{18}$ for multiple case studies was used. Within-case and between-case analyses were performed with the care process for patients with STEMI in the PCI centres as the unit of analysis. First, all interview transcripts were reviewed line-by-line and split into three predetermined process steps: (1) first (para)medical contact in person to PCI decision; (2a) activation of the catheterisation room; and (2b) PCI decision to the start of the PCI procedure. Process steps $2 \mathrm{a}$ and $2 \mathrm{~b}$ occur simultaneously. Use of additional strategies to reduce the treatment delay is mentioned separately. Next, the care process per PCI centre, taking into account different patient routings, was described in detail (within-case analysis). The textual and graphical descriptions (swim lane charts) were linked by using similar annotation. An example of a swim lane chart describing the general process of prehospital care is presented in online supplementary figure S1. Differences in the logistic processes of PCI centres with the European guideline-recommended process and with one another were identified by comparing the textual and graphic process descriptions (between-case analysis).

Factors accelerating the care process but not pertaining to it were identified by reviewing all transcripts line by line. All issues related to accelerating factors as indicated by the participants were extracted from the transcripts and coded by means of open coding (content analysis). Subsequently, text fragments with similar open codes were bundled in an axial coding process and their contents were analysed inductively to reveal the core categories of factors accelerating the care processes.

After conducting and transcribing 12 interviews (covering all PCI centres), JT performed an interim analysis in which additional care providers in the process and potential gaps in the descriptions of the process per PCI centre were identified. To improve the reliability of the coding scheme, three interviews were coded independently by a second researcher (IvdW) and differences in the coding were discussed until consensus was reached. As a result, small modifications to the definitions in the coding scheme were made accordingly.

All transcripts were coded using the computer program ATLAS.ti (V.5.2, ATLAS.ti Scientific Software Development GmbH).

\section{Verification}

The textual and graphical process descriptions of each PCI centre were sent back to the participating interventional cardiologists for verification. All interventional cardiologists responded, resulting in minor changes in the process descriptions.

\section{Ethical approval}

This study was approved by the Medical Ethical Committee of the VU University Medical Center. All participants were informed about the study goals and data processing, and written consent for study participation and audio recording of the interview was obtained. Data were stored on a password-protected network drive of the VU University Medical Center, to which only the researchers had access. Codes were assigned to participants and centres for privacy purposes. Additionally, all transcripts were anonymised by removing all names of people, centres and geographical locations.

\section{RESULTS}

From six PCI centres, 25 care providers were interviewed (table 1). One centre did not respond to the invitation and could therefore not be included. Moreover, to verify the general prehospital care process, two general practitioners and three cardiologists from referring hospitals were invited for participation, of whom one general practitioner and two cardiologists participated. This resulted in a total of 28 interviews with a mean duration of 45 min (range 23-68 min).

\section{Differences from the European guideline-recommended process and between $\mathrm{PCl}$ centres}

Differences between the care processes of individual PCI centres, the European guideline-recommended process and between PCI centres could be allocated to one of the process steps below. Differences between PCI centres are summarised in the lower part of figure 1. Illustrative quotes are presented in online supplementary table S2 and referred to in the text. The letters between brackets in the text correspond to the letters for the PCI centres presented in table 1 .

\section{First ( para)medical contact in person to $\mathrm{PCl}$ decision}

The prehospital care processes of the PCI centres differed from the European guideline-recommended process on one aspect. The European guideline-recommended process assumes that patients arrive at the catheterisation room from the emergency medical services. In this study, additional patient routings were identified in which patients were already admitted to a hospital department, for example, the surgery department, or presented to emergency departments of PCI centres or non-PCI centres. The cooperation with the ambulance services was highly protocolled, while the cooperation with the general practitioners, emergency departments, other hospital departments or referring hospitals was much less protocolled. In case patients go to the general practitioner, no ECG is performed and the emergency medical services are contacted immediately. 
Table 1 Characteristics of participating $\mathrm{PCl}$ centres and interview participants

\begin{tabular}{|c|c|c|c|c|c|c|}
\hline Hospital & $\mathbf{a}$ & b & c & d & e & $\mathbf{f}$ \\
\hline Type of hospital & Teaching & Teaching & Academic & Teaching & Teaching & Teaching \\
\hline Provision of thoracic surgery & No & Yes & Yes & No & Yes & Yes \\
\hline Number of catheterisation rooms (primarily for $\mathrm{PCl}$ ) & $2(1)$ & 5 (3 or 4$)$ & $3(2)$ & $2(1)$ & $5(2$ or 3$)$ & $3(2)$ \\
\hline Number of primary $\mathrm{PCI}$ procedures per year & $300-400$ & $>500$ & $400-500$ & $<300$ & $>500$ & $>500$ \\
\hline Interventional cardiologist & $x$ & $x$ & $x$ & $x$ & $\mathrm{x}$ & $x$ \\
\hline Cardiology resident & & $x$ & $\mathrm{x}$ & & $x$ & $x$ \\
\hline Catheterisation room nurse & $x$ & $x$ & $x$ & $x$ & $x$ & $x$ \\
\hline Cardiac care unit nurse & $x$ & & $\mathrm{x}$ & $x$ & & \\
\hline Ambulance nurse/medical manager & $x$ & $x$ & $x$ & $x$ & $x$ & $x$ \\
\hline Referring cardiologist* & & & & $x$ & & $x$ \\
\hline General practitioner $^{*}$ & & & $x$ & & & \\
\hline
\end{tabular}

${ }^{*}$ Participants in Italic for verification.

$\mathrm{PCl}$, percutaneous coronary intervention.

Between PCI centres, several differences were found. When patients are announced at the PCI centre by the emergency medical services, the emergency department or referring hospitals, there can be uncertainty or ambiguity about the working diagnosis. However, additional diagnostic tests might result in a prolonged treatment delay. The PCI centres differed in the way they dealt with the trade-off between diagnostic certainty and speed. Some PCI centres had dedicated facilities to receive prehospital ECGs transmitted from the ambulance, followed by a telephone call $(\mathrm{a}, \mathrm{b}, \mathrm{c}, \mathrm{f})$. This provided the possibility for a coronary care unit nurse (a) or cardiology resident (b,c,f) to confirm the diagnosis. After hours, in one centre (c) all ECGs were additionally forwarded to the interventional cardiologist on call for review. Other centres had no dedicated facilities for ECG reception, but occasionally ECGs were received through (protected) mobile phone applications (d,e). One PCI centre was able to receive ECGs; however, not all ambulance service providers in the region were equipped to transmit ECGs (b), leading to variation within the region.

In case there were facilities for transmitting and receiving ECGs (a,b,c,f), different criteria were used for the decision to employ these facilities. Some PCI centres required all ECGs to be transmitted $(\mathrm{a}, \mathrm{c})$, while others only required ambiguous ECGs to be transmitted (b,f), thereby minimising the number of consultations for unambiguous diagnoses (quote 1). In the PCI centres where only the ambiguous ECGs were transmitted or which had no dedicated facilities to receive ECGs, the patient was always accepted for angiography and PCI without additional diagnostic testing or consultation when an ambulance nurse indicated with certainty that the patient had an STEMI (b,d,e,f; quote 2). As a result, no ECGs were transmitted and the PCI centre was only contacted by telephone to convey additional information about the patient. In one centre (a), the decision to send a patient for PCI was made by a dedicated coronary care unit nurse, while in another centre (c) a cardiology resident made the decision. In case the diagnosis was uncertain, a cardiology resident or interventional cardiologist could be consulted, resulting in additional discussion before the patient was accepted.

\section{Activation of the catheterisation room}

In all PCI centres included in this study, the catheterisation room was activated by a dedicated care coordinator at the PCI centre. The profession of the care coordinator differed between PCI centres. In one centre (a), the coordinator was a dedicated nurse from the coronary care unit both during office hours and after hours. The nurse had the autonomy to read the ECG and activate the catheterisation room without additional consultation of a cardiologist or cardiology resident (quote 3). In three centres, during office hours a cardiologist activated the catheterisation room while after hours a cardiology resident $(b, f)$ or coronary care unit nurse (d) was responsible. In two other centres, the cardiology resident was always responsible for catheterisation room activation (c, e). However, in one of these centres (c), a coronary care unit nurse was contacted first through a landline, who subsequently transferred the call to the cardiology resident, resulting in an additional process step. The difference in profession of the care coordinator indicates different choices between speed (a readily available nurse) and reliability of the diagnosis (a more difficult to reach cardiologist).

During office hours, the catheterisation room staff was already present as they performed elective PCI procedures and thus the catheterisation room systems were already operational. When an incoming patient required primary PCI, ongoing or planned elective procedures in one of the catheterisation rooms were cancelled and rescheduled to free the room for the incoming patient. $\mathrm{Up}$ to the point where a sheath or guidewires were inserted, patients undergoing elective procedures were removed from the catheterisation room to speed up its availability.

After hours, the catheterisation room staff on call (interventional cardiologist and multiple catheterisation room nurses) were generally not present at the centres. 
There were two exceptions. One centre required all staff on call who had to travel over $20 \mathrm{~min}$ to the centre to stay overnight (f), while at another centre some cardiologists stayed overnight voluntarily (e). The catheterisation room staff staying overnight at the centre initiated catheterisation room preparations immediately after announcement of the patient, thereby optimally using the transport time of the patient. At two centres $(\mathrm{a}, \mathrm{b})$, preparation of the catheterisation room was initiated by a coronary care nurse working the evening or night shift while the catheterisation room staff and patient were en route to the centre (quote 4 ).

An addition to the European guideline-recommended process was found in the way the catheterisation room staff was contacted after hours. The interventional cardiologist and catheterisation room nurses were called by the care coordinator or doorman to present to the PCI centre within 20-30 min. None of the centres used a single-call page system but contacted the staff by (mobile) telephone instead, enabling immediate confirmation. To start the procedure as soon as possible, only the interventional cardiologist and at least one catheterisation nurse had to be present.

\section{$\mathrm{PCl}$ decision to the start of the $\mathrm{PCl}$ procedure}

As recommended in the European guideline, the emergency department was bypassed on arrival at the hospital and the patients were transported directly to the catheterisation room on the ambulance stretcher. When the catheterisation room was not available yet, patients were accommodated in a holding area near the catheterisation room or in the coronary care unit. In contrast to the European guideline-recommended process, in one centre the patients made an additional stop at the coronary care unit (d; quote 5), from where a coronary care unit nurse transported the patient to the catheterisation room. The nurse stayed to assist in the procedure and transported the patient back to the coronary care unit to facilitate continuity of care.

In case the catheterisation room was not ready on arrival of the patient at the PCI centre, several strategies for accommodating the patient were identified. Some PCI centres had a dedicated holding area for patients awaiting catheterisation at the catheterisation room complex on the ambulance stretcher $(\mathrm{a}, \mathrm{b})$, while others admitted patients at the coronary care unit $(c, f, e)$. One centre sporadically set up these patients at another catheterisation room, so that only the staff had to change rooms (b).

\section{Monitoring of delays}

An additional strategy in the European guidelinerecommended process was to monitor the treatment delays and identify variation. However, only one PCI centre used this strategy (a), though all centres recorded information about the treatment delay to report on national quality indicators.

\section{Accelerating factors}

Several factors not pertaining to the process were identified by the healthcare providers as accelerating the care process. These factors were categorised as patient, healthcare provider, interprovider and PCI centre characteristics and are summarised in the upper part of figure 1 .

\section{Patient characteristics}

Participants mentioned that the clarity of the signs and symptoms experienced by the patient determines the way patients interpret their symptoms, and the care provider that they contact. The participants indicated that bypassing the general practitioner and calling the emergency medical services directly accelerated the care process. Furthermore, in contacting a care provider, the patient's assertiveness can influence the priority that he/she is being given, for example, by insisting that the symptoms are severe. Additionally, when patients are haemodynamically stable, fewer resources, that is, equipment, care providers (eg, anaesthesiologist) and diagnostic procedures (eg, an ultrasound) are required, which accelerates the process.

\section{Healthcare provider characteristics}

A working diagnosis is the starting point for the PCI centre to initiate preparations for receiving a patient with STEMI. Therefore, the working diagnosis needs to be made prehospital by an experienced and qualified diagnostician in order to make a reliable diagnosis to ensure activation of the catheterisation room without further diagnostic testing (quote 6). Also, participants indicated that in the communication with the PCI centre, it is vital that the diagnostician is assertive in case he/she is certain of the working diagnosis of STEMI in order to avoid additional transfer of information and unnecessary consultations. Consequently, in some centres, a cardiology resident had to consult the interventional cardiologist, in which the same factors of experience, qualification and assertiveness are important for the cardiology resident, thereby limiting the potential delay caused by the additional consultations.

\section{Interprovider characteristics}

In case a working diagnosis is made, the communication between the ambulance crew and the PCI centre needs to be clear, quick, unambiguous and direct. Participants stressed that this will minimise discussion about the diagnosis, resulting in a shorter treatment delay. One important requirement is trust in the diagnosis made by the ambulance nurse or other diagnostician. When trust in the diagnosis is high, no further discussion is needed and preparations for the PCI procedure can be initiated (quote 7).

However, doubt about the working diagnosis can result in additional intercaregiver consultations in which the diagnosis is discussed, potentially delaying catheterisation room activation and patient transport. Therefore, 


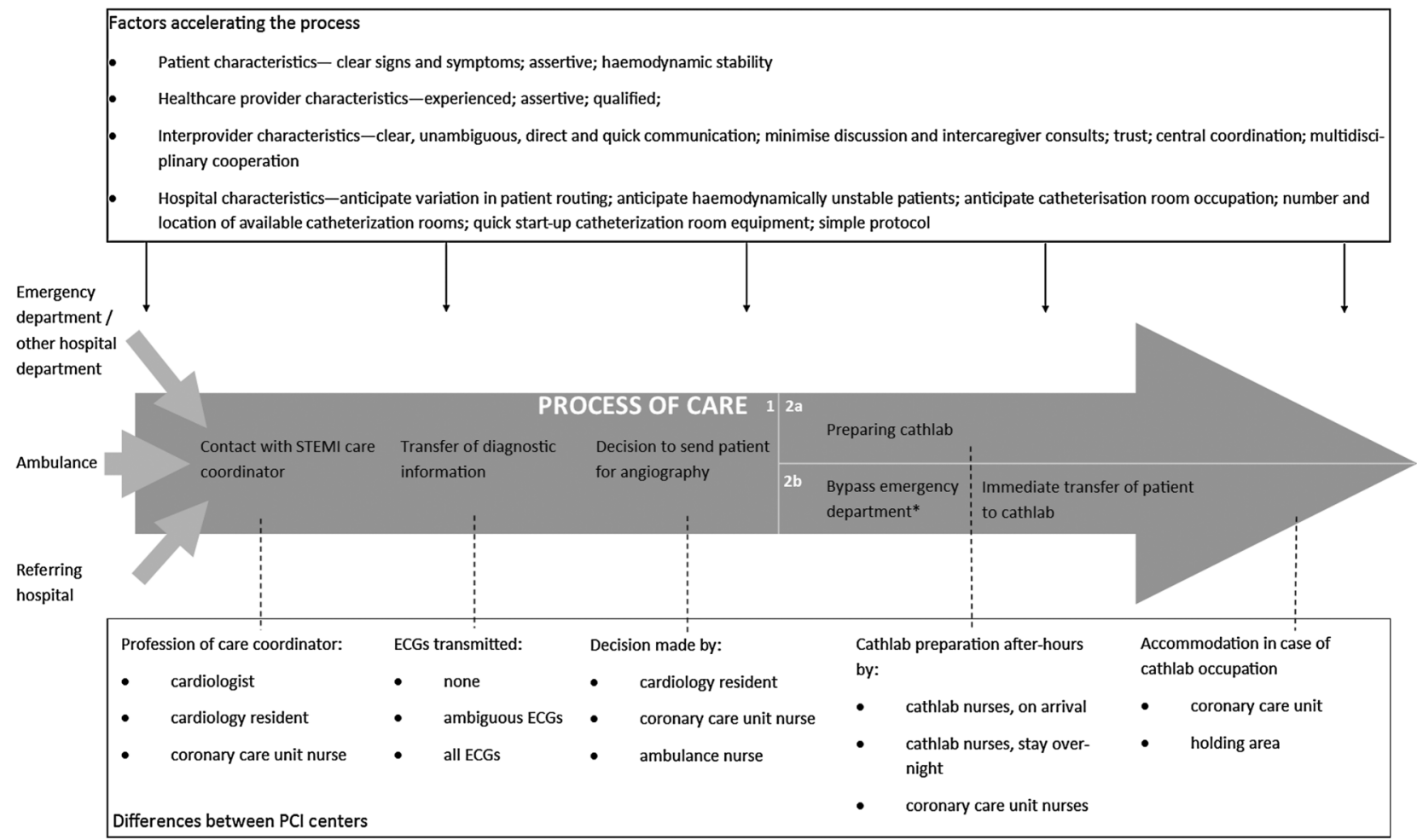

* Irrelevant in case the emergency department was the first medical contact for the patient); 1 : from first (para)medical contact to PCl decision; 2a: activation of the cathlab; 2b: PCl decision to start of the $\mathrm{PCl}$ procedure

Figure $1 \mathrm{~A}$ model of variation and accelerating factors in the process of acute care chain of patients with STEMI going for primary PCI. PCl, percutaneous coronary intervention; STEMI, ST segment elevation myocardial infarction.

multidisciplinary cooperation in minimising doubt about the diagnosis and agreeing on an acceptable level of uncertainty in the region of a PCI centre is deemed as an important factor accelerating the process in the long run.

\section{$\mathrm{PCl}$ centre characteristics}

The catheterisation room location should ideally be near the ambulance entrance, the emergency department and the coronary care unit of the PCI centre to limit transfer times. In addition, having multiple catheterisation rooms increases the chances that one is available. Having catheterisation room equipment with a short start-up time as well as having a simple protocol describing everyone's responsibilities can reduce the time to prepare the catheterisation room.

Anticipation on different patient routings and characteristics was identified by the participants as important for optimising the process speed. For example, some PCI centres anticipate transporting haemodynamically unstable patients to the catheterisation room as soon as the patient is stable enough. The cardiologist goes to the emergency department immediately after prehospital notification to provide care and to remind other care providers that the patient needs to go to the catheterisation room as soon as possible. At the catheterisation room, specialised staff (eg, anaesthesiologist) with advanced equipment are waiting to take care of the patient. PCI centres also anticipated catheterisation room occupancy by having a dedicated holding area near the catheterisation room.

\section{DISCUSSION}

In this study, the care processes in multiple PCI centres for patients with STEMI going for primary PCI in the Netherlands were compared with the European guideline-recommended process and to one another. In addition, factors accelerating the process were determined. The PCI centres differed from the European guideline-recommended process on additional, unavoidable patient routings and monitoring delays. Differences between PCI centres included the way diagnostic information was communicated and having personnel ready on-site to immediately prepare the catheterisation room. Accelerating factors included the patients' assertiveness, trust in the diagnosis of colleagues and avoiding intercaregiver consultations and discussion.

In all PCI centres, the emergency medical services bypassed the emergency department on arrival at the hospital. This is in accordance with previous study results, in which direct transport to the catheterisation room was identified as a time-saving strategy. ${ }^{19}$ However, the procedures for patients entering the PCI centres through alternative routings were less protocolised. Therefore, it is important that in optimising the care process, PCI centres take into account all patient 
routings in shared protocols and infrastructure for all involved care providers in their region. Continuously updating and disseminating the guideline-recommended process enables individual PCI centres to compare their own process with the synthesis of best practices. Additional improvements in comparison to our model can be shared within and between STEMI care networks. ${ }^{20}$

In the design of the acute care process for patients with STEMI, PCI centres appeared to seek a balance between accuracy of the diagnosis and speed of the process. While some centres chose a system in which the ambulance nurse decided to send the patient for PCI, other centres chose a system in which the ECG first had to be evaluated by a coronary care unit nurse, cardiology resident or interventional cardiologist. This additional verification of the diagnosis may result in a prolonged treatment delay, while on the other hand it may prevent unnecessary catheterisation room activations. Previous studies indicated proficient interpretation of ECGs by emergency medical services staff, ${ }^{21}{ }^{22}$ indicating that transmittal of the ECG might not be required. ${ }^{23}$ However, transmitting the ECG allows for comparison to previous ECGs, thereby potentially minimising unnecessary catheterisation room activation. ${ }^{24}$ An optimal configuration of the care process may be identified by means of computer simulation, in which the effect of an altered configuration on the treatment delay and the diagnostic certainty can be evaluated. Computer simulations have been applied in decreasing patient wait times at the emergency department, ${ }^{25}$ and could also be of value in optimising the treatment delay.

Although driving times are prolonged in Denmark, a higher number of procedures per operator were linked with improved patient outcomes. ${ }^{26}$

The optimal level of centralisation of primary PCI procedures within countries remains unclear. Although driving times can be shorter in a more decentralised approach, a more centralised approach can result in a higher number of PCI procedures per operator. Both aspects of care have been linked to improved patient outcomes, ${ }^{2}{ }^{26}$ but they appear mutually exclusive. To gain insight into an optimal centralisation strategy, a natural experiment can be used, for example, by comparing patient outcomes in the Netherlands and Denmark. Denmark, with 5.6 million inhabitants in $43000 \mathrm{~km}^{2}$ and $\sim 2700$ primary PCI procedures each year, ${ }^{12}$ is comparable to the Netherlands on the terrain, infrastructure and guideline recommendations. ${ }^{27-30}$ The major difference appears to be the number of PCI centres providing primary PCI procedures: 30 PCI centres for 16.8 million inhabitants (560 000 inhabitants per PCI centre) in the Netherlands versus 4 PCI centres for 5.6 million inhabitants (1.4 million inhabitants per PCI centre) in Denmark. In addition, the organisation of the emergency medical services (25 regions in the Netherlands vs 5 regions in Denmark) is also more centralised. It is unclear to what extent this further centralisation of STEMI care influences patient outcomes. It would therefore be recommendable to compare patient outcomes between the Dutch and Danish systems in order to identify an optimal level of centralisation.

The accelerating factors could be organised in four categories: patient, provider, interprovider and PCI centre characteristics. In reducing the treatment delay, it appears crucial to redesign the process while taking into account factors that may influence the speed of the process. Although not all factors can be influenced directly by care providers, anticipating situations in which these factors play a role may help to minimise treatment delays, for example, having an adapted process for haemodynamically unstable patients. In addition, both provider and interprovider characteristics are mentioned as accelerating the process. These categories justify multidisciplinary training within a region in order to align the views of all involved care providers, including general practitioners, referring hospitals and emergency department staff. Taking into account these accelerating factors may further decrease the treatment delay in the long run.

The differences in care processes and accelerating factors are presented as independent factors, while in real life they may be inter-related. For example, when an interventional cardiologist trusts the diagnosis made by an ambulance nurse or coronary care unit nurse, no further discussion is required. This trust can in turn be influenced by the experience and qualification of the diagnostician and by the clarity of the patients' signs and symptoms. Low trust in the diagnosis might result in additional discussion or an additional stop at the emergency department for further diagnostic testing, prolonging the treatment delay. Consequently, in optimising the process, it is important to consider both process steps and accelerating factors.

\section{Study limitations}

The study results should be interpreted taking potential limitations into consideration. Personal views and experiences of participants may have affected the reliability of data collected by means of interviews. The accelerating factors were perceived by the care providers and not tested quantitatively and should therefore be interpreted with caution. To increase the reliability of the data, a variety of PCI centres and care providers per PCI centre and its region were included.

The data were collected in a single country, which may have influenced the usability of the study findings for other countries. However, the care of patients with STEMI has been standardised in international cardiology guidelines and primary PCI is the reperfusion method of choice in many countries. ${ }^{10}$ Therefore, the findings of this study can be useful internationally, though differences in the national protocols or accelerating factors may occur.

In this study, no professionals from the emergency department were interviewed. This was because 
cardiologists and cardiology residents were closely involved in the care for patients with a suspected cardiac disease at the emergency department. In addition, in the Netherlands, only a very small number of patients arrive through the emergency department. Strategies to optimise care for patients with STEMI that focus on the emergency department will therefore have limited effect in reducing the average treatment delay in a hospital. However, a part of the process was not directly explored in this study, which could have affected the reliability of the reported results.

Finally, we were unable to link the processes identified per PCI centre in this study to time intervals or patient outcomes because of size differences between the regions in which centres were located which may affect the treatment delay. As a consequence, it cannot be determined to what degree the reported process differences and accelerating factors accounted for a shorter treatment delay. However, the overall treatment delay in the participating centres was relatively short ${ }^{6}$ compared with other studies, and multiple time-saving strategies as identified in previous best practices were applied in all PCI centres, for example, prehospital diagnosis and bypassing the emergency department. ${ }^{9}$

\section{CONCLUSIONS}

Several differences in the current acute care process for patients with STEMI in comparison to the European guideline-recommended process and between PCI centres were found. These differences potentially affect the treatment delay, indicating room for further improvement. Hospitals can learn from each other's process designs by identifying and sharing best practices. The results of this study therefore facilitate future quality improvement efforts and research that may eventually reduce the treatment delay of patients with STEMI.

Acknowledgements The authors would like to thank all participating hospitals and care providers for their cooperation.

Contributors JT designed the study; collected, analysed and interpreted the data; and wrote the initial draft of the paper. MCdB and IvdW helped analyse and interpret the data and revised subsequent versions of the paper. MCdB and CW helped design the study and revised subsequent versions of the paper.

Funding This work was supported by the Dutch Ministry of Health, Welfare and Sport.

Competing interests None declared.

Ethics approval Medical Ethical Committee of the VU University Medical Center.

Provenance and peer review Not commissioned; externally peer reviewed.

Data sharing statement No additional data are available.

Open Access This is an Open Access article distributed in accordance with the Creative Commons Attribution Non Commercial (CC BY-NC 4.0) license, which permits others to distribute, remix, adapt, build upon this work noncommercially, and license their derivative works on different terms, provided the original work is properly cited and the use is non-commercial. See: http:// creativecommons.org/licenses/by-nc/4.0/

\section{REFERENCES}

1. Terkelsen CJ, Sørensen JT, Maeng M, et al. System delay and mortality among patients with STEMI treated with primary percutaneous coronary intervention. JAMA 2010;304:763-71.

2. Boersma E, Primary Coronary Angioplasty vs. Thrombolysis Group. Does time matter? A pooled analysis of randomized clinical trials comparing primary percutaneous coronary intervention and in-hospital fibrinolysis in acute myocardial infarction patients. Eur Heart J 2006;27:779-88.

3. Steg PG, James SK, Atar D, et al. ESC Guidelines for the management of acute myocardial infarction in patients presenting with ST-segment elevation. Eur Heart J 2012;33:2569-619.

4. O'Gara PT, Kushner FG, Ascheim DD, et al., American College of Cardiology Foundation; American Heart Association Task Force on Practice Guidelines; American College of Emergency Physicians, et al. 2013 ACCF/AHA guideline for the management of ST-elevation myocardial infarction: executive summary: a report of the American College of Cardiology Foundation/American Heart Association Task Force on Practice Guidelines: developed in collaboration with the American College of Emergency Physicians and Society for Cardiovascular Angiography and Interventions. Catheter Cardiovasc Interv 2013;82:E1-27.

5. Blankenship JC, Skelding KA, Scott TD, et al. Predictors of reperfusion delay in patients with acute myocardial infarction undergoing primary percutaneous coronary intervention from the HORIZONS-AMI trial. Am J Cardiol 2010;106:1527-33.

6. Tra J, van der Wulp I, de Bruijne MC, et al. Exploring the treatment delay in the care of patients with ST-elevation myocardial infarction undergoing acute percutaneous coronary intervention: a cross-sectional study. BMC Health Serv Res 2015;15:340.

7. Peterson MC, Syndergaard T, Bowler J, et al. A systematic review of factors predicting door to balloon time in ST-segment elevation myocardial infarction treated with percutaneous intervention. Int J Cardiol 2012;157:8-23.

8. De Luca G, Suryapranata H, Ottervanger JP, et al. Time delay to treatment and mortality in primary angioplasty for acute myocardial infarction: every minute of delay counts. Circulation 2004;109:1223-5.

9. de Winter RJ. Measuring hospital performance in the management of acute coronary syndromes. Neth Heart $J$ 2014;22:344-5.

10. Kristensen SD, Laut KG, Fajadet J, et al., European Association for Percutaneous Cardiovascular Interventions. Reperfusion therapy for ST elevation acute myocardial infarction 2010/2011: current status in 37 ESC countries. Eur Heart $J$ 2014;35:1957-70.

11. Yin RK. Case study research: design and methods. 4th edn, SAGE, 2009.

12. Widimsky $\mathrm{P}$, Wijns $\mathrm{W}$, Fajadet J, et al. Reperfusion therapy for ST elevation acute myocardial infarction in Europe: description of the current situation in 30 countries. Eur Heart J 2010;31:943-57.

13. Dutch College of General Practitioners. NHG standaard acuut coronair syndroom [NHG standard acute coronary syndrome], 2013.

14. Regional ambulance services. Ambulance care in the Netherlands, 2013. https://www.ambulancezorg.nl/download/downloads/1755/ ambulance-care-in-the-netherlands-2013.pdf (accessed online Jan 2017).

15. Ambulancezorg Nederland. Landelijk protocol ambulancezorg versie 7.2 [National protocol ambulance care version 7.2], 2011.

16. Health Council of the Netherlands. A solid foundation is a must! Quality at a basic accident and emergency department within a regional network. The Hague: Health Council of the Netherlands, 2012.

17. Tra J, Engel J, van der Wulp I, et al. Monitoring guideline adherence in the management of acute coronary syndrome in hospitals: design of a multicentre study. Neth Heart J 2014;22:346-53.

18. Miles M, Huberman A. Qualitative data analysis: an expanded sourcebook. 2nd edn. Thousand Oaks, CA, USA: SAGE, 1994.

19. Dorsch MF, Greenwood JP, Priestley C, et al. Direct ambulance admission to the cardiac catheterization laboratory significantly reduces door-to-balloon times in primary percutaneous coronary intervention. Am Heart J 2008;155:1054-8.

20. Jollis JG, Granger CB, Henry TD, et al. Systems of care for ST-segment-elevation myocardial infarction: a report From the American Heart Association's Mission: Lifeline. Circ Cardiovasc Qual Outcomes 2012;5:423-8.

21. Cantor WJ, Hoogeveen $\mathrm{P}$, Robert A, et al. Prehospital diagnosis and triage of ST-elevation myocardial infarction by paramedics without advanced care training. Am Heart J 2012;164:201-6.

22. Feldman JA, Brinsfield K, Bernard S, et al. Real-time paramedic compared with blinded physician identification of ST-segment elevation myocardial infarction: results of an observational study. Am J Emerg Med 2005;23:443-8. 
23. Strauss DG, Sprague PQ, Underhill K, et al. Paramedic transtelephonic communication to cardiologist of clinical and electrocardiographic assessment for rapid reperfusion of ST-elevation myocardial infarction. J Electrocardiol 2007;40:265-70

24. Wong CK. Minimizing false activation of cath lab for STEMI-a realistic goal? Int J Cardiol 2014;172:e91-3.

25. Shim SJ, Kumar A. Simulation for emergency care process reengineering in hospitals. Bus Process Manage J 2010;16: 795-805.

26. Strom JB, Wimmer NJ, Wasfy JH, et al. Association between operator procedure volume and patient outcomes in percutaneous coronary intervention: a systematic review and meta-analysis. Circ Cardiovasc Qual Outcomes 2014;7:560-6.
27. Clemmensen $\mathrm{P}$, Schoos MM, Lindholm MG, et al. Pre-hospital diagnosis and transfer of patients with acute myocardial infarctiona decade long experience from one of Europe's largest STEMI networks. J Electrocardiol 2013;46:546-52.

28. Lassen JF, Bøtker HE, Terkelsen CJ. Timely and optimal treatment of patients with STEMI. Nat Rev Cardiol 2013;10:41-8.

29. Schoos MM, Sejersten M, Hvelplund A, et al. Reperfusion delay in patients treated with primary percutaneous coronary intervention: insight from a real world Danish ST-segment elevation myocardial infarction population in the era of telemedicine. Eur Heart $J$ Acute Cardiovasc Care 2012;1:200-9.

30. Rasmussen MB, Frost L, Stengaard C, et al. Diagnostic performance and system delay using telemedicine for prehospital diagnosis in triaging and treatment of STEMI. Heart 2014;100:711-15. 